\title{
A Rare Painful Mediopatellar Plica and Cord-Like Structure Complex in the Knee Joint
}

Yukio Nakamura ${ }^{1,2^{*}}$, Seneki Kobayashi ${ }^{3}$ and Hiroyuki Kato ${ }^{2}$

${ }^{1}$ Department of Orthopaedic Surgery, Showa Inan General Hospital, Akaho, Komagane, Japan

${ }^{2}$ Department of Orthopaedic Surgery, Shinshu University School of Medicine, Asahi, Matsumoto, Japan

${ }^{3}$ Department of Orthopaedic Surgery, Suwa Red Cross Hospital, Kogandori, Suwa, Japan

"Corresponding author: Yukio Nakamura, Department of Orthopaedic Surgery, Shinshu University School of Medicine, Asahi 3-1-1, Matsumoto 360-8621, Japan, Tel: +81-263-37-2659; Fax: +81-263-35-8844; E-mail: yxn14@aol.jp

Rec date: Dec 29, 2015; Acc date: Feb 05, 2016; Pub date: Feb 08, 2016

Copyright: (c) 2016 Nakamura Y, et al. This is an open-access article distributed under the terms of the Creative Commons Attribution License, which permits unrestricted use, distribution, and reproduction in any medium, provided the original author and source are credited.

\begin{abstract}
Anterior knee pain is a frequent, yet relevant, symptom since mediopatellar plicae are found in approximately $20 \%$ of normal knees. We report the rare case of a painful mediopatellar plica and cord-like structure complex covering the suprapatellar pouch in the knee joint. The patient was a 13-year-old girl who complained of left anterior knee pain with restricted range of motion that had started 1 year earlier. She underwent arthroscopic treatment after unsuccessful conservative therapy. Intraoperative findings included a Sakakibara classification Type B mediopatellar plica and band-like structure complex located in the suprapatellar pouch of the knee. As Type A and B plicae are generally asymptomatic, the cause of the pain in this case was presumed to be impingement of the mediopatellar plica by the cord-like band. The plica and band-like structure complex was resected and ablated by arthroscopy. The patient was pain-free several months postoperatively and could resume normal school life. Since she was an adolescent in her growth phase, it is possible that joint growth resulted in tension on the remnant of the plica connected with the band-like structure in the suprapatellar pouch, which then intruded into the medial aspect of the patellofemoral joint and resulted in her symptoms.
\end{abstract}

Keywords: Knee; Mediopatellar plica; Pain

\section{Case Presentation}

We report a rare case based on the intraoperative finding of an abnormal cord under the patella. A 13-year-old girl visited our institution with a 1-year history of left anterior knee pain and difficulty in daily walking and running. She had no history of joint injury. Left knee range of motion (ROM) was $5^{\circ}$ of extension to $120^{\circ}$ of flexion, which had become restricted due to pain. Swelling or inflammation were not evident. Clinical tests for knee stability were comparable with those of the contralateral side, and the McMurray test for meniscal tear was negative. Magnetic resonance image (MRI) examination and plain radiographs of the affected knee did not reveal any notable abnormalities, nor did blood tests indicate signs of inflammation (data not shown). The patient commenced a conservative treatment regimen of joint-strengthening exercises, but no improvements were seen. Six months after presentation, she underwent arthroscopy under general anesthesia. There were no remarkable findings in the menisci, cartilage, anterior cruciate ligament, or posterior cruciate ligament. However, a mediopatellar plica classified as Sakakibara Type B was observed in the suprapatellar pouch along with a band-like structure connected to the plica and covering the patellofemoral joint [1-5] (Figure 1a). The complex was removed using basket forceps, an electrothermal probe, and an arthroscopic shaver (Figure 1b). At follow-up 2 months postoperatively, the patient reported no knee joint pain, and knee joint ROM was fully restored.

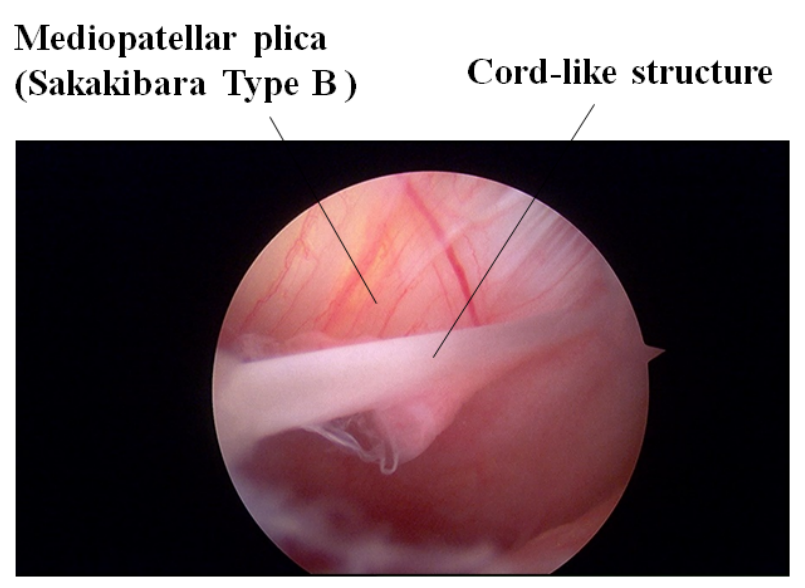

Figure (1a): Arthroscopic image of a Sakakibara Type B mediopatellar plica in the suprapatellar pouch. A cord-like structure connected to the plica and covering the patellofemoral joint is present. 


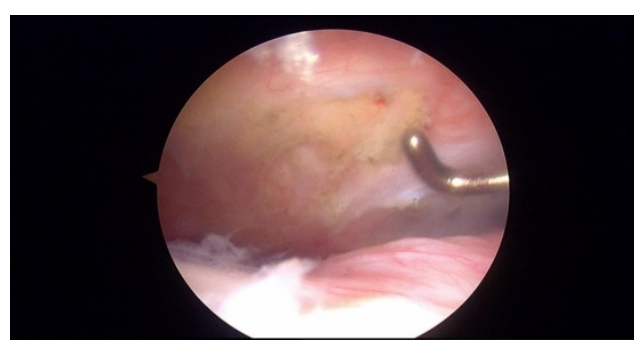

Figure 1(b): The complex was removed using basket forceps, an electro-thermal probe, and an arthroscopic shaver.

\section{Discussion}

The suprapatellar plica is a remnant of the embryologic synovial membrane between the suprapatellar pouch and the knee joint. This report describes the clinical outcome of a 13-year-old girl with a mediopatellar plica combined with a band-like structure in the suprapatellar pouch. To the best of our knowledge, there has been no such report of this combination leading to restricted ROM and knee joint pain (Figure 2).

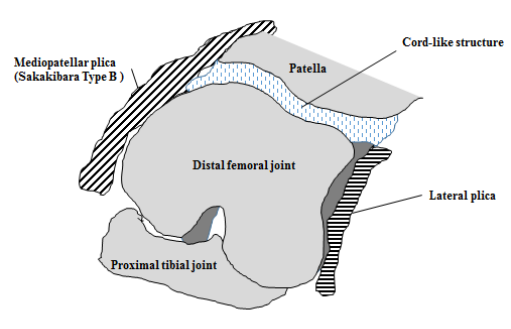

Figure 2: Schematic image of the Sakakibara classification Type B mediopatellar plica, lateral plica, and cord-like structure complex located in the suprapatellar pouch of the knee.

The suprapatellar plica has been described as a synovial fold in the suprapatellar pouch of the knee joint that is present in approximately $20 \%$ of the population. This fold can become symptomatic after injury and cause symptoms similar to other common internal derangements of the knee [6]. Regardless of their location, plicae vary widely in terms of size, shape, and configuration. The medial suprapatellar plica manifests as a crescent-shaped fold originating beneath the quadriceps tendon and extending to the medial wall of the joint. It courses obliquely downward relative to the patella, attaching distally to the synovial membrane covering the infrapatellar fat pad [7].

Nakayama et al. [8] examined the incidence and severity of medial plicae during arthroscopic surgery in a large cohort study of Japanese patients (mean age: 35 years) based on the Sakakibara classification system $[5,8]$. The incidence of medial plica was $79.9 \%$. Type A grading was the most frequently encountered, followed next by Types B, C, and D. Generally, whereas Type C and D plicae are known to cause knee joint pain, Type A and B plicae are considered to cause no or mild symptoms [8]. Since the patient's plica had a shelf-like appearance in the mediopatellar region, it was classified as Type B. However, she suffered from joint pain while walking and running in daily life activities and was unresponsive to conservative treatment.

The anatomy of the medial suprapatellar plica and medial synovial shelf has been studied arthroscopically in a report of 500 knees, in which the medical suprapatellar plica was found to extend up to one third of the way across the suprapatellar pouch in $64.2 \%$ of knees, between one and two thirds in $4 \%$, and two thirds or more in $31.6 \%$. The medial synovial shelf was absent or vestigial in $36 \%$ of knees and broader than $1 \mathrm{~cm}$ in $13.2 \%$. Although there was no association between large plicae and large shelves, a shelf was absent significantly more often in knees with a narrow plica [9].

Pathologic synovial plicae are uncommon but can cause a variety of clinical manifestations. Pekmezci et al. described the case of a pathologic plica involving a check-valve mechanism [10]. Yilmaz et al. reported that retinacular bands located beneath plicae on the anteromedial aspect of the knee in patients with plica syndrome played a role in the symptomatology and pathophysiology of plica syndrome and that their excision improved outcome [11]. In this case, similar band-like structures that resembled retinacular bands might have caused the anterior knee pain, as Yilmaz et al. have remarked [11]. Since the patient was presumably in her adolescent growth phase, joint growth may have resulted in tension on the remnant of the plica connected with the band-like structure in the suprapatellar pouch, which then intruded into the medial aspect of the patellofemoral joint and resulted in her symptoms.

Lastly, Adachi et al. have reported on mobility improvement in the patella after resection of complete-type suprapatellar plica by arthroscopy. They speculated that the main cause of the anterior knee pain was cartilaginous damage of the patella and trochlea that might have been accelerated by the existence of the suprapatellar plica, thus reducing the mobility of the patella [12]. However, we detected no alterations in knee joint cartilage in preoperative MRI scans or during surgical observation.

\section{Summary}

Surgical removal of a unique mediopatellar plica and band-like structure complex provided immediate pain resolution in an adolescent with knee pain and ROM restriction.

\section{References}

1. Ehlinger M, Moser T, Adam P, Simon P, Bonnomet F (2009) Complete suprapatellar plica presenting like a tumor. Orthop Traumatol Surg Res 95: 447-450.

2. McCunniff PT, Anthony CA, McDermott SE, Duchman KR, Albright JP (2013) Arthroscopic validation of the clinical exam for the detection of the symptomatic synovial-plical complex by relief from surgical excision. Iowa Orthop J 33: 78-83.

3. Irha E, Vrdoljak J (2003) Medial synovial plica syndrome of the knee: a diagnostic pitfall in adolescent athletes. J Pediatr Orthop B 12: 44-48.

4. Stubbings N, Smith T2 (2014) Diagnostic test accuracy of clinical and radiological assessments for medial patella plica syndrome: a systematic review and meta-analysis. Knee 21: 486-490.

5. Sakakibara J (1976) Arthroscopic study on linos band (plica synovia is mediopatellaris). J Jpn Orthop Assoc. 50: 513-522.

6. Apple JS, Martinez S, Hardaker WT, Daffner RH, Gehweiler JA (1982) Synovial plicae of the knee. Skeletal Radiol 7: 251-254.

7. Hardaker WT, Whipple TL, Bassett FH 3rd (1980) Diagnosis and treatment of the plica syndrome of the knee. J Bone Joint Surg Am 62: 221-225. 
Citation: Nakamura Y, Kobayashi S, Kato H (2016) A Rare Painful Mediopatellar Plica and Cord-Like Structure Complex in the Knee Joint. J Clin Case Rep 6: 705. doi:0.4172/2165-7920.1000705

Page 3 of 3

8. Nakayama A, Sugita T, Aizawa T, Takahashi A, Honma T (2011) Incidence of medial plica in ,889 knee joints in the Japanese population. Arthroscopy 27: 1523-1527.

9. Dandy DJ (1990) Anatomy of the medial suprapatellar plica and medial synovial shelf. Arthroscopy 6: 79-85.

10. Pekmezci M, Atay OA, Kerimoglu U, Aydingoz U, Tetik O,et al. (2006) A complete supra-patellar plica with an unusual presentation. Knee Surg Sports Traumatol Arthrosc. 14: 872-874.
11. Yilmaz C, Golpinar A, Vurucu A, Ozturk H, Eskandari MM (2005) Retinacular band excision improves outcome in treatment of plica syndrome. Int Orthop 29: 291-295.

12. Adachi N, Ochi M, Uchio Y, Kawasaki K, Yamasaki K (2004) The complete type of suprapatellar plica in aprofessional baseball pitcher: consideration of a cause of anterior knee pain. Arthroscopy 20: 987-991. 